\title{
Large Print Desktop-Publishing by PC for the Partially Sighted
}

\author{
Ulrich Zeun
}

Department of Special Education, University of Dortmund, Germany

\begin{abstract}
This paper emphasizes the need of large print textbooks for partially sighted readers as a alternative reading medium. Research showed that there are almost no production facilities for large print, too little large print titles, and widely spread photocopying does not fit the perceptual needs of low vision people in all aspects. First experience of a large print project working with regular PC-equipment are stated, and guidelines are given concerning technical equipment, large print layout and typography. Though image editing is a more time-consuming and and sophisticated job than text editing, adapted pictures should be included in large print books.
\end{abstract}

\section{Introduction - Starting point of research}

Though electronic texts become more and more available, screen reading is not the ideal reading situation for the partially sighted. This is due to physiological conditions as well as disadvantages of screen enlargement software compared to black print reading techniques such as browsing, skipping from leaf to leaf, getting a full page overview etc. - Software enlarged screens only show a fraction of the regular 25 line and 80 columns screen.

In spite of all discussion, whether reading large print or reading normal print with optical aides is preferrable [6], large print still has its justification. This is even more true, if the large print is adapted didactically. Enlarged photocopies bear disadvantages: Flaws in print such as hard-to-read typefaces, a too small spacing, blurred and broken strokes of letters cannot be altered. On the contrary, flaws are sometimes emphazised [9]. Moreover, photocopying often results in unhandy and bulky formats [7]. Colour copiers are still expensive as well as color copies made in a copy shop. So, colour copies are seldomly done. Colour information is lost. 
This was the starting point of our idea to find out how large print including coloured graphics can be adapted near to optimally for low visioners by taking advantage of modern PC-technology for large print production. Our project is named "Large Print Service for the Partially Sighted" (German abbreviation is GrUsS = Großdruck-Umsetzungsservice für Sehbehinderte).

Two questionnaires one for teachers for low vision students, the other for publishing houses, and specialized adaptation services for the visually impaired should reveal, whether and if yes how large print is made. Here are some results of the situation in German speaking coumtries:

\section{Schools for the partially sighted / Teachers' methods:}

- teachers mainly use photocopiers, but colour copiers are only available in 5 of 45 German schools for visually impaired pupils;

- computer und printers are used only by a few teachers;

- a service producing large print materials for partially sighted students not only would relieve the teacher from time-consuming material adaptation but is also welcome by a majority of the teachers.

\section{Publishing houses - special services}

- there are only some publishing houses that have book series in large print. But the type size is seldomly more than 12 points ${ }^{1)}$, a size I consider too small. Larger type sizes can rather be found amongst school book publishers, especially for primary grade readers;

- their large print books cover only novels but no school or university textbooks;

- only two specialized institutions produce fitting large print: the "Schweizerische Bibliothek für Blinde und Sehbehinderte in $\mathrm{Zu}$ rich" (Swiss Library for the Blind and Partially Sighted) enlarges print with a photocopier, but also produces textbooks, whereas the "Deutsche Zentralbücherei in Leipzig" (German Central Library for the Blind) started a computer aided reproduction, but scientific books are not included.

- existing large print material are one time productions, and partly out-of-date,

1) I do not distinguish here betwenn American and Western-European point measure. 
- existing and newly produced large prints do not include coloured graphics.

This big lack of textbooks for partially sighted students needs improvement by establishing a production and dessimination center. A concept has already been elaborated for supplying study material. This concept was developed by the visaully impaired students themselves and their "Federal-wide Workgroup on Access to Literature for Visually Impaired Students" [2].

\section{Remark:}

Again, according to the publishers demands for camera-ready typesetting, this article is reproduced contrary to some typographical needs we consider to be adequate for the partially sighted. A large print sample is added at the end of the article.

\section{Adapted books and demand for large print material}

Who are the addressees?

There are two groups that should be supplied with large print books: pupils and university students.

The project GrUsS has not yet adapted university textbooks, because the partially sighted at the University of Dortmund have not demanded for large print material or contacted us, partly because they had yet not known about the GrUsS project or hesitated to demand large print as long as they can manage reading somehow - not necesarily better. We did adapt material for partially sighted in the past, though. Adapted materials were conference brochures and some on-campus material, such as newsletters or the menu-card of the cantine [12].

Examining study material, however, shows that scientific books comprise rather less than too many graphics and colored images compared to modern school books. Thus, the problems we will mention below take place less often with university textbooks. Experiences we gathered from adapting school books can be transferred better to university books than vice versa. 
What textbooks are needed?

Teachers called for dictionaries, vocabulary lists, and indexes. Above all, a demand for lavishly pictured textbooks in geography, mathematics or foreign languages were uttered. Maps are needed, too. Those latter books and texts can all be counted among books with colored illustrations which can not be copied in the school without loss of quality and didactical expression.

\section{Technical aspects}

What technical PC equipment do we use?

The project is equipped with the following hard- and software:

Input devices

- a greyscale flatbed scanner (Ricoh RS632, 300 dpi) for scanning texts and line graphics,

- a colour flatbed scanner (Microtek Scanmaker II Xe, 400 dpi or more) for scanning images,

- a graphics tablet (Genius),

- keyboard and mouse

- triple-speed CD-ROM drive ${ }^{*}$

Processing and editing

- two PCs (80386 and $80486^{*} \mathrm{cpu}$ )

- an OCR/ICR software (Omnipage Professional 5.0),

- an image processing software (PhotoStyler 2.0) ${ }^{*}$,

- other picture and painting software on trial (e.g. CorelDraw, Windows-Paintbrush, Harvard Graphics),

- word processing / DTP software (Word for DOS 5.5, Ami Professional 2.0, PageMaker $5.0^{*}$ ).

Storing, back-up

- gigabyte hard disk drives, ${ }^{*}$

- gigabyte magneto-optical disk drive,"

- gigabyte streamer system.*

Output and telecommunication

- a PostScript laser printer (NEC Silentwriter, 300 dpi), 
- a colour PostScript inkjet printer capable of DIN A3 outputs (IBM Lexmark 4079 PS),

- a 24 pins matrix colour printer capable of DIN A3 outputs (NEC P70c),

- a high-speed modem for transferring formatted data to schools and others ${ }^{*}$.

From my point of view, it is important to use regular PC-products (low and medium cost) to find out, how the average user, teacher or smaller production facility can carry out production processes on their own. Therefore, using the Apple Macintosh, as many professional publishers and DTP services do, was left out for the Apple system is not as wide spread as IBM-compatible PC systems in schools and among teachers or visually impaired users. The same holds true for $T_{E} X$. It is only used at universities and by its members. We make use of the standardised PostScript language and compatible printers to make large print outputs.

Results about shortcomings of the used system can be expected. Additions will be and were already neccessary (marked with an astericks in the equipment list above). High end setting and print machines are surely rather commendable for bigger production centres.

\section{First experiences}

As coloured textbooks were demanded, we had to use a colour scanner. First experiences - we started in 1993 - revealed that immense storing capacities are needed for colour scans. An image can use up to twenty megabytes or more depending on its original size and scanning resolution. To set no limits, we raised funds for purchasing large storing media in 1994.

Handling pictures within texts made it also neccessary to buy a so-called desktop publishing software (i.e. PageMaker). This or other similar software is more suitable for importing graphics, rotating and editing images within the text layout than word processing software running under Windows. However the DTP software has other cutbacks compared to editors, such as no footnote management or no editor for scientific formulae (we use the Ami Pro math editor; the WinWord math editor can be used with PageMaker). 


\section{Perceptual and diddactical aspects}

The following remarks concern perceptual aspects on typography for partially sighted readers, and didactical needs of an adapted layout. I will differentiate betwenn mere text, scientific formulae or graphic symbols, and graphics.

\section{Text}

We did some research on typographical layout for the partially sighted by

a) studying relevant literature $[1,2,10,11]$,

b) applying a perceptual test on partially sighted readers - readers were given text samples of different layout, typefaces and with different point sizes -, and

c) our first experiences during adapting material.

From that, we drew some guidelines for large print typography and layout. To number all, would make up a seperate paper. Only some old and new guidelines shall be mentioned here (for further details feel free to contact me). Some of the guidelines are contrary to typographical rules often stated for normal visioners $[2,8]$.

1) Sans-serif typefaces are all in all better readable for partially sighted readers than serif fonts - an average of 18pt typesize suits many low vison readers.

2) For low visioners with problems of resolution sans-serif typefaces should be used that are highly distinguishable in terms of letter differentiation. Typefaces for reading beginners, for example, show characteristic differences between the small "l" and the capital "I", or the capital "J".

3) Head column titles should be contrasted from the text body through bigger and bold letters, thus allowing a good orientation within a book.

4) The paging should be at the outside top margin of the page and be set in huge pointsizes so that the partially sighted reader can perceive it at first glance even from a wider distance. Moreover doing this, the reader does not need to open the book fully while browsing for a certain page. 
5) Scientific textbooks and those used in mainstreaming programmes must have references to the original print page numbers. This enables the reader to quote correctly or to refer to the page to be read. We mark page number by special non used characters (black triangles) within the text as well as on the botton margin of the page set in larger print and in lined frames.

6) A line spacing of five typografical points is advantageous (compared to 2 or 3 points).

7) The standard paper format (i.e. DIN A4 or letter) should be used for reasons of handling and weight.

To comply with these guidelines for large print formatting, you can use so-called style-sheets which every versatile word processor offers, and thus allows quick formatting. This function defines the format of text, paragraph, and page layout.

\section{Formulae and graphic symbols}

As semigraphical and mathematical symbols cannot be scanned and recognized, they must be typed anew. We use Ami Professional's math editor to set the scientific figures of a mathematical textbook (Ami uses $\mathrm{T}_{\mathrm{E}} \mathrm{X}$ for encodeing the figures!).

It is neccessary to set mathematical symbols in larger type sizes. This is due to two reasons. First, math symbols are not as uniformally characteristic as letters. This worsenes perception. Second, keeping the regular large print point size (average of $18 \mathrm{pt}$.) would let numbers of fractions or exponents appear much too small. The base size therefore must be increased. Similar results are to be expected for musical notation. Though there was yet no demand for musical textbooks, a larger typesize for the musical symbol set should be used. Presumably, a musical editor will have to be used for it.

\section{Coloured Images}

Problems of adapting material can rather occur with images than with text. Black and white or line art graphics can still be scanned quite easily, and edited afterwards. In these cases, it is most of all important to simplify the graphics structure. A process we know from preparing tactile graphics for the blind. Simplifying can sometimes mean 
to draw the graphic newly. Editing coloured images, however, is a much more sophisticated job. Time for training and handling the image editing software is higher. Working on the images is also more time-consuming than it is when editing line art.

What adaptation must be used upon coloured graphics? Coloured graphics sometimes must be enlarged, simplified, - labelled anew, changed in colour contrast.

We use two mehtods of enlarging. Either you can keep the regular portrait format of a DIN A4 page, or you can print the picture in landscape format. Landscape paging enables a higher enlargemnet.

Results are: pictures or photos that are clearly structured and are unambigious need to be enlarged less (e.g. a agricultural countryside photo with clear-cut meadows and fields of green, yellow and brown). Complex images or photographs, however, demand higher enlargement (e.g. a bird's view of an industrial town). Enlargement will partly depend on the perceptual skill and his or her environmental pre-experiences of the partially sighted reader. The first picture could be printed regularly in portrait format, a landscape enlargement was appropriate for the second one. The low resolution of the landscape enlarged photograph - due to the printers standard resolution of only $360 \mathrm{dpi}$ - was not found to be inadequate by the partially sighted reader.

Mere enlargement of graphics and images will not suffice. Typefaces of images are partly serif fonts, partly set on coloured backgrounds which lead to low reading contrast. Adaptation is a must (but is not done by adapting services shrinking from the plus of editing work). So images must be labelled anew. Labelling should mostly be done with vector orientated painting software for pixel-based painting software reproduces zigzag outlines of the letters. For example we used CorelDraw to reset words rather than using the image editing software PhotoStyler. To change reading contrast either the background colour has to be altered to a lighter hue (e.g. light yellow or light blue) or the text is set into a white framed box. Negative contrast, i.e. white labeling on black background frames means optimal contrast. Positive and negative contrast can be used altenatively for different categories (e.g. country names vs. capital town names on a map). 
Colour contrast must be adapted especially for readers with colour perception problems or colour blind people. Adjactent planes should be coloured far from each other on terms of satuaration, e.g. deep blue contrasting to light yellow. After this principle, adjactent colours reproduced or seen in greyscale will also be differntiable for the colour blind. In case more than two planes meet, optimal greyscale and colour contrast for all adjactent planes may be impossible. Colour changes can be made as long as the colours do not carry certain meanings as they do on topographical maps, or as long as they do not signalize meanings (e.g. red stands for danger, red stop sign) [4]. Natural colours within photographs must not be estranged, but you can change a dark blue of the sky to a lighter hue or a dark brown of an earth layer within a sectional view to beige. Colour editing tools such as the "colour pick" (eyedropping tool) or the "bucket fill" to catch a hue and to colour a whole plane enable us to do these changes.

Contour lines (black and/or white) between planes can effect in better defferentiation. Simple line art paintings such as wood engravings make use of these perceptual advantages. That is why they are often preferred as illustrations in large print books (e.g. books from the Library in Leipzig). We have yet not tested perceptual pros and cons of hatched planes.

\section{Conclusion and outlook}

We have seen that large print production especially of textbooks with coloured images is neglected. There are only a few services that already provide some books. Our project on PC-based large print editing shows that though adaptation is quite time consuming - but this is also true for braille adaptations - it can be done with low-cost or medium cost DTP systems. These experiences must be carried on further. Efforts to supply large print must be intensified. Adaptation guidelines and methods should be elaborated further. Perhaps some adapting processes such as colour changes could be automatically done by a software still to be developed.

I would be thankful for every idea or cooperating partner. From my personal point of view as a partially sighted reader, I would welcome 
every institutional effort to produce large print as an alternaive reading medium to optical aids.

contact: Universität Dortmund - FB 13 - GrUsS -

Ulrich Zeun - Emil-Figge-Straße 50 - 44221 Dortmund phone: 0231/755-4579 - FAX: 0231 / 7554558

\section{Sources and Reading proposals:}

[1] Barraga, Natalie C./ Morris, Jane E.: (1978) Program to Develop Effeciency in Visual Functioning (Source Book on Low Vision), Ed.: American Printing House for the Blind, Louisville, .

[2] Baumann, Hans D./ Klein, Manfred: (1990/2) DesktopPublishing - Typografie und Layout (Seiten gestalten am PC für Einsteiger und Profis), Niedernhausen.

[3] BAG-L (Bundesarbeitsgemeinschaft zur Literaturbeschaffung für sehgeschädigte Studierende): unpublished concept paper.

[4] Boldt, Werner: (1969) "Über die Bedeutung von Lehr-, Arbeitsund Hilfsmitteln bei der Erziehung und Rehabilitation Blinder und Sehbehinderter", in: die aula, pp. 156-161.

[5] Burtt, Harold, E.: (1949) "Typography and Readability", in: The Sight-Saving-Review, 19. vol, No. 3, pp. 147-157.

[6] Corn, Anne L./ Ryser, Gail R.: (1989) “Access to Print for Students with Low Vision", in: Journal of Visual Impairment and Blindness, 83. vol, pp. $340-349$.

[7] Fonda, Gerald: (1965) Management of the patient with subnormal vision, St. Louis.

[8] Hochuli, Jan: (1987) Das Detail in der Typografie; Wilmington

[9] Shaw, Alison: (1969) Print for Partial Sight, Ed.: The Library Association, London.

[10] Tanner, Margarete: (1971) "Schriftgut für sehbehinderte Kinder", in: Zeitschrift für das Blinden- und Sehbehindertenbildungswesen, 91. vol, pp. $175-181$.

[11] Tinker, Miles A. (1965) Bases for Effective Reading, Minnesota, . [12] Zeun, Ulrich: (1993) Aufbereitung hochschulinterner Materialien für sehgeschädigte - Abschlußbericht, Dortmund

Credits: our thanks for donations and support go to "Sieglinde Hildebrandt-Stiftung”, Aldus Software, Fa. Frank Audiodata 


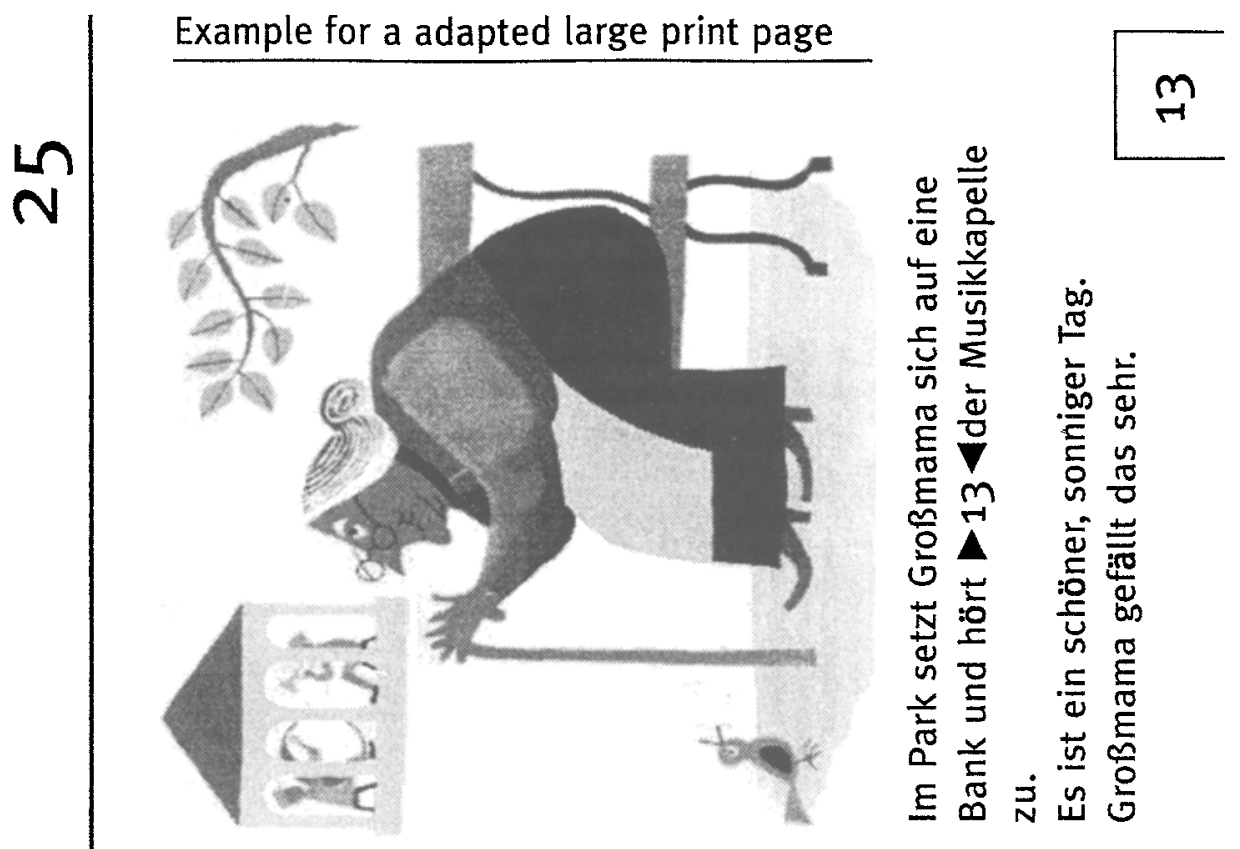

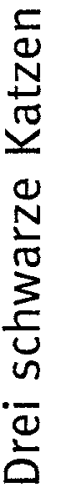

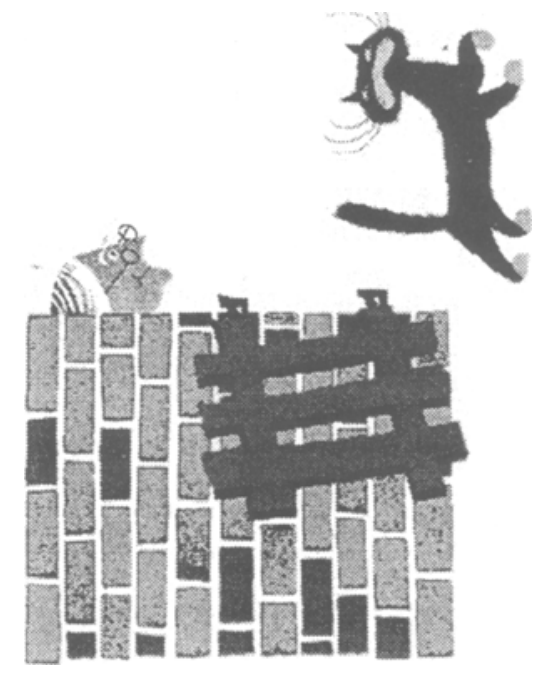

